\title{
Rhabdomyolysis in a Teenage Boy: A Case Report
}

\author{
Jobn M. Sauret, MD, and Carlos Roberto Jaén, MD, PbD
}

Rhabdomyolysis is a potentially lethal syndrome with a wide range of metabolic and clinical aspects. It is caused by skeletal muscle injury that results in the release of myoglobin and other cellular contents into the circulatory system. ${ }^{1}$ Multiple factors predispose someone to this illness. Preventive measures can avoid the onset of this syndrome, and a timely diagnosis and proper management can avert life-threatening complications. This report describes a case of exertional rhabdomyolysis in a patient who sought treatment for bilateral leg pain.

\section{Case Report}

A 19-year-old male university student complained to his local campus infirmary of bilateral leg pain and dark-colored urine. This condition occurred 4 hours after completing a vigorous physical workout. He was trying out for the varsity rowing team, which involved 90 vertical jumps, 2 miles of running, and leg presses consisting of four sets of 20 repetitions. During the past several months he had not exercised regularly. Two days before reporting to the infirmary, he had cold-like symptoms (ie, nasal congestion, clear nasal discharge, sore throat). The evening before admission, he had consumed 10 beers. He had no notable medical or surgical history and was not taking any medications, and he reported no illicit drug use and was a nonsmoker.

Intravenous hydration was started, and the patient was transferred to the local emergency department from the university infirmary because of his extreme pain and trouble walking. At admission his respiratory rate was $16 / \mathrm{min}$, pulse 84 beats per minute, blood pressure $132 / 72 \mathrm{~mm} \mathrm{Hg}$, and temperature $98.4^{\circ} \mathrm{F}$. He was alert, responsive, and cooperative. His conjunctiva were anicteric and non-

Submitted, revised, 27 January 1999.

From the Department of Family Medicine (JMS, CRJ), School of Medicine and Biomedical Sciences, SUNY at Buffalo. Address reprint requests to John $M$ Sauret, $M D$, Department of Family Medicine, State University of New York at Buffalo Office of Research and Development, 462 Grider St, Buffalo, NY 14215. injected, his oral mucosa was dry, his lungs were clear to auscultation, and his heart sounds were normal. There was no lymphadenopathy, and findings of the abdominal examination were benign. $\mathrm{He}$ had considerable pain, swelling, and tenseness over the quadriceps muscle compartment that kept him from flexing at his knee or being able to bear weight. His peripheral pulses were palpable and his neurologic findings were unremarkable. A laboratory analysis showed a creatine phosphokinase (CPK) of 213,700 U/L, which was repeated for verification. A urinalysis of a dark-colored specimen showed blood $(3+)$ (free hemoglobin or myoglobin), no bacteria, no red blood cells, no urobilinogen, $\mathrm{pH} 6.5$, and a urine myoglobin of $>2500$ $\mathrm{ng} / \mathrm{mL}$. Other pertinent laboratory results at admission were as follows: serum alanine aminotransferase (ALT) $490 \mathrm{U} / \mathrm{L}$, serum aspartate aminotransferase (AST) $2560 \mathrm{U} / \mathrm{L}$, lactate dehydrogenase $3450 \mathrm{U} / \mathrm{L}$, sodium $139 \mathrm{mEq} / \mathrm{L}$, potassium 4.3 $\mathrm{mEq} / \mathrm{L}$, blood urea nitrogen $11 \mathrm{mg} / \mathrm{dL}$, creatinine $0.7 \mathrm{mg} / \mathrm{dL}$, glucose $100 \mathrm{mg} / \mathrm{dL}$, calcium $9.0 \mathrm{mg} /$ $\mathrm{dL}$, and phosphorus $3.8 \mathrm{mg} / \mathrm{dL}$ (Table 1).

Rhabdomyolysis was suspected, and vigorous intravenous forced diuresis was initiated before he was transferred to a regular medical floor. His urine was alkalinized by adding 1 ampule of sodium bicarbonate $(\mathrm{NaHCO} 3)$ to each liter of $0.9 \%$ sodium chloride $(\mathrm{NaCl})$. Throughout the 7-day hospitalization, serial CPK levels were measured, and electrolytes, calcium, and phosphate remained within normal limits. Blood in the urine was used as a surrogate marker for myoglobin. By the seventh day of admission the total CPK dropped to 1806 $\mathrm{U} / \mathrm{L}$, his electrolytes remained stable, and his myoglobinuria resolved, as did his previously elevated liver enzyme levels.

Physical therapy was initiated on the third day of admission. On a daily basis the patient underwent range of motion exercises on his lower extremities and was subjected to weight-bearing exercises. On discharge from the hospital, he was able to bear his 
Table 1. Laboratory Results by Day of Hospitalization.

\begin{tabular}{|c|c|c|c|c|c|c|c|c|c|c|}
\hline Day & $\begin{array}{l}\text { CPK } \\
\mathrm{U} / \mathrm{L}\end{array}$ & $\begin{array}{l}\text { Sodium } \\
\mathrm{mEq} / \mathrm{L}\end{array}$ & $\begin{array}{l}\text { Potassium } \\
\mathrm{mEq} / \mathrm{L}\end{array}$ & $\begin{array}{c}\text { Creatinine } \\
\mathrm{mg} / \mathrm{dL}\end{array}$ & $\begin{array}{c}\text { BUN } \\
\mathrm{mg} / \mathrm{dL}\end{array}$ & $\begin{array}{l}\text { Calcium } \\
\mathrm{mg} / \mathrm{dL}\end{array}$ & $\begin{array}{c}\text { Phosphorus } \\
\mathrm{mg} / \mathrm{dL}\end{array}$ & $\begin{array}{c}\text { Bicarbonate } \\
\mathrm{mEq} / \mathrm{L}\end{array}$ & $\begin{array}{l}\mathrm{ALT} \\
\mathrm{U} / \mathrm{L}\end{array}$ & $\begin{array}{l}\text { AST } \\
\mathrm{U} / \mathrm{L}\end{array}$ \\
\hline 1 & 213,700 & 143 & 4.3 & 0.7 & 11 & 9.0 & 3.8 & 32 & 490 & 2560 \\
\hline 2 & 190,200 & 140 & 4.6 & 0.8 & 10 & 8.6 & 4.4 & 30 & 478 & 2310 \\
\hline 3 & 97,300 & 143 & 4.4 & 0.6 & 9 & 8.8 & 3.3 & 31 & 499 & 2453 \\
\hline 4 & 46,000 & 141 & 4.2 & 0.7 & 8 & 8.8 & 4.7 & 32 & 479 & 1890 \\
\hline 5 & 11,020 & 142 & 4.0 & 0.7 & 9 & 8.9 & 4.0 & 32 & 423 & 1281 \\
\hline 6 & 3,799 & 142 & 3.9 & 0.7 & 8 & 8.7 & 4.2 & 28 & 334 & 613 \\
\hline 7 & 1,806 & 142 & 3.9 & 0.5 & 10 & 8.7 & 4.0 & 31 & 198 & 222 \\
\hline
\end{tabular}

CPK_creatine phosphokinase, BUN_blood urea nitrogen, ALT_alanine aminotransferase, AST—aspartate aminotransferase.

full weight and perform knee flexion to 115 degrees. Two weeks after discharge he was doing well and received a forearm ischemic test and a muscle biopsy, the results of which were within normal limits. This forearm ischemic test is done by acquiring baseline serum lactic acid and ammonia levels from the antecubital vein. Subsequently, a sphygmomanometer cuff around the upper arm is inflated to $>200 \mathrm{~mm} \mathrm{Hg}$. This pressure is greater than the systolic pressure, thus creating an ischemic condition at which time that forearm will be fatigued by hand-grip exercises. The cuff is removed and serial lactic acid and ammonia levels are measured. $^{2}$ If there is minimal or no rise in the lactic acid level, then McArdle disease (myophosphorylase deficiency) can be diagnosed. If the ammonia levels also show slow or no rise, then myoadenylate deaminase deficiency can be diagnosed.

\section{Discussion}

This patient manifested a case of acute exertional rhabdomyolysis. According to the National Center for Health Statistics, approximately 26,000 reported cases of rhabdomyolysis are reported yearly in the United States. ${ }^{3}$ The following factors are included in a relatively long list of causes of rhabdomyolysis: (1) direct injury to skeletal muscle, as from trauma, burns, infections, influenza, coxsackievirus, and toxins such as alcohol; (2) decreased oxygen delivery to skeletal muscle, as from carbon monoxide poisoning, compression syndrome, and arterial occlusion; (3) increased energy consumption, as from extreme exertion, malignant hyperthermia, heat stroke, and such drugs as cocaine and amphetamines; and (4) decreased energy storage and utilization, as from diabetic ketoacidosis, nonketotic hyperosmolar coma, myoedema, and hypothermia. ${ }^{4}$

The clinical course of rhabdomyolysis will vary depending on the underlying cause. Early recognition is important so that severe complications, such as renal failure, electrolyte abnormalities, disseminated intravascular coagulation, and compartment compression syndrome, can be prevented. ${ }^{1}$ Acute renal failure complicates up to $15 \%$ of cases of rhabdomyolysis. ${ }^{5}$ The release of large amounts of myoglobin has been implicated in the development of acute tubular necrosis and ultimately renal failure, although there is no statistical correlation between level of serum CPK and serum or urine myoglobin. ${ }^{6}$ Urine alkalinization and forced diuresis will prevent tubular pigment reabsorption, helping to preserve renal function and also prevent other life-threatening complications. In one study of exercise-induced rhabdomyolysis, no patients developed acute renal failure after forced bicarbonate diuresis within the first 6 hours of admission. ${ }^{7}$

Within the first 24 hours of admission, this patient received $10 \mathrm{~L}$ of $0.9 \%$ sodium chloride, each liter containing 1 ampule of sodium bicarbonate to achieve appropriate urine alkalinity $(\mathrm{pH}>6.5)$ and forced diuresis. If fluid loading is not sufficient to achieve diuresis, an intravenous dose of either furosemide (40 $\mathrm{mg}$.) or mannitol ( $25 \mathrm{~g}$ ) may be used as an adjuvant to assist with urine output. Severe hyperkalemia secondary to the massive injury to skeletal muscle and subsequent leakage of potassium into the circulation is also a potential complication. Toxicity can be potentiated by the release of large amounts of phosphates from the muscle cell, causing a relative hypocalcemia. Serum calcium and phosphorus levels should be measured on a daily 
basis. Hepatic dysfunction occurs in approximately $25 \%$ of patients with rhabdomyolysis. ${ }^{8}$ Proteases released from injured muscle might be implicated in this hepatic derangement, which is reversible. The pathogenesis is not well-defined and might be multifactored. ${ }^{8}$

Compartment syndrome of the thigh is believed to be rare because the thigh has a large potential space to allow for swelling and increased interstitial pressure before circulation is endangered. ${ }^{9}$ Clinical signs of this syndrome are swelling, tenseness, and pain. Nerve deficits, mainly sensory, are more important findings in compartment syndrome. In our case, the patient had considerable pain and swelling but no neurologic abnormalities. Peripheral pulses can still be palpated in this syndrome because compartment pressures might not be high enough to occlude major arteries. The clinical examination is sufficient to diagnose compartment syndrome in a patient who is awake and cooperative, and this syndrome was excluded in this patient. When the classic signs and symptoms are unobtainable in patients who are unconscious or not able to cooperate (ie, due to mental illness) with the physical examination, quantitative measurements of compartment pressures assume an increasingly important diagnostic role. ${ }^{10}$ Measuring compartment pressures involves placing a catheter into the compartment and connecting it to a pressure monitor. Decompressive fasciotomy should be considered for pressures $>30 \mathrm{~mm} \mathrm{Hg} .{ }^{10}$ In this case, fasciotomy was not indicated.

There are several measures to prevent the recurrence of acute exertional rhabdomyolysis. This patient had no history of alcohol abuse, and the amount he consumed was unlikely to have played a role in his condition. Even so, however, he was advised to eliminate alcohol consumption, especially during an exercise program. Rhabdomyolysis resulting from alcohol consumption usually occurs in chronic alcoholics who have episodes of extreme drinking. Metabolic myopathies place persons at high risk of recurrence. Ischemic forearm testing, which has shown to be an invaluable tool for screening of patients with exercise-related muscular complaints, ${ }^{2}$ should be performed to rule out glycogen storage diseases and myoadenylate deaminase deficiency. This patient did not exercise regularly and engaged in a rigorous physical activity, which ultimately caused this condition.
Table 2. Rhabdomyolysis: Diagnosis, Management, and Prevention.

\begin{tabular}{ll}
\hline Diagnosis & History of current illness \\
& Physical examination \\
& Elevated creatine phosphokinase \\
Management & Forced diuresis \\
& Diuretics as adjuvant therapy \\
& Urine alkalinization \\
& Check blood urea nitrogen, creatinine \\
& Monitor renal and electrolyte status (calcium \\
& and potassium) \\
& Rule out compartment syndrome \\
& Rule out disseminated intravascular \\
& coagulation \\
& Physical therapy \\
& Graded exercise programs \\
& Appropriate hydration \\
& Avoid strenuous activity in hot and humid \\
climates & Alcohol cessation advice \\
Frevention & Forearm ischemic test \\
Muscle biopsy & Rule out metabolic myopathies
\end{tabular}

Prevention of rhabdomyolysis is prudent. $\mathrm{Pa}$ tient education and teaching a proper exercise program that gradually increases the intensity of the workout, along with appropriate hydration before, during, and after exercise, are likely the most important measures. In addition, strenuous physical activity in hot, humid climates and alcohol consumption should be avoided. In the setting of rhabdomyolysis, appropriate patient evaluation and care are vital to avoid potentially lethal complications (Table 2). Management must involve attempting to preserve renal function. Hydration, forced diuresis, and electrolyte monitoring are essential.

Eileen Sauret and Andrew Danzo have provided contributions to this article.

\section{References}

1. Line RL, Rust GS. Acute exertional rhabdomyolysis. Am Fam Physician 1995;52:502-6.

2. Sinkeler $\mathrm{S}$, Wevers RA, Joosten $\mathrm{EM}$ et al. Improvement of screening in exertional myalgia with a standardized ischemic forearm test. Muscle Nerve 1986; 9:731-7.

3. Graves EJ, Gillium BS. National discharge hospital survey. Detailed diagnoses and procedures. Vital and Health Statistics, no 13. 1997. [DHHS publication no PHS 98-1799.]

4. Cheng J, Feinfeld D. Rhabdomyolysis and myoglo- 
binuria In: Conn RB, Snyder JW, editors. Current diagnosis. Philadelphia: WB Saunders,1997:978-9.

5. Ward MM. Factors predictive of acute renal failure in rhabdomyolysis. Arch Intern Med 1988;148:1553-7.

6. Feinfeld DA, Cheng JT, Beysolow TD, Briscoe AM. A prospective study of urine and serum myoglobin levels in patients with acute rhabdomyolysis. Clin Nephrol 1992;38:193-5.

7. Sinert R, Kohl L, Rainone T, Scalea T. Exerciseinduced rhabdomyolysis. Ann Emerg Med 1994;23: 1301-6.
8. Akmal M, Massry SG. Reversible hepatic dysfunction associated with rhabdomyolysis. Am J Nephrol 1990;10:49-52.

9. Kahan JS, McClellan RT, Burton DS. Acute bilateral compartment syndrome of the thigh induced by exercise. A case report. J Bone Joint Surg Am 1994;76: $1068-71$.

10. Schwartz JT Jr, Brumback RJ, Lakatos R, Poka A, Bathon GH, Burgess AR. Acute compartment syndrome of the thigh. A spectrum of injury. J Bone Joint Surg Am 1989;71:392-400. 